Scientific Journal of Silesian University of Technology. Series Transport Zeszyty Naukowe Politechniki Śląskiej. Seria Transport

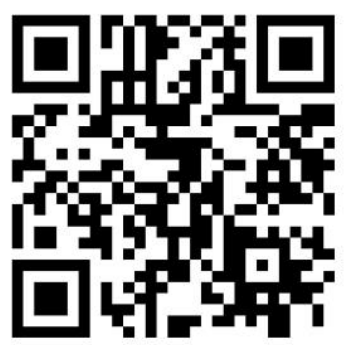

Volume 114

2022

p-ISSN: 0209-3324

e-ISSN: 2450-1549

DOI: https://doi.org/10.20858/sjsutst.2022.114.13

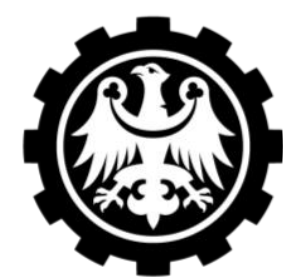

Silesian

University of Technology

Journal homepage: http://sjsutst.polsl.pl

Article citation information:

Storani, F., Di Pace, R., De Luca, Memoli, S. Calibration and validation of a macroscopic traffic flow model based on platoon dispersion and queue propagation. Scientific Journal of Silesian University of Technology. Series Transport. 2022, 114, 155-167. ISSN: 0209-3324. DOI: https://doi.org/10.20858/sjsutst.2022.114.13.

Facundo STORANI ${ }^{1}$, Roberta DI PACE ${ }^{2}$, Stefano DE LUCA ${ }^{3}$, Silvio MEMOLI ${ }^{4}$

\title{
CALIBRATION AND VALIDATION OF A MACROSCOPIC TRAFFIC FLOW MODEL BASED ON PLATOON DISPERSION AND QUEUE PROPAGATION
}

Summary. This paper proposes a preliminary calibration and validation of a macroscopic traffic flow model for signalised junctions. In fact, on the network signal setting design problem, a reliable modelling approach must be adopted to acknowledge the traffic flow effects, considering two phenomena: queue dispersion and spillback. The proposed model is an extension of the space-time discrete Cell Transmission Model (CTM), which can simulate dispersion and horizontal queue. This preliminary calibration and validation use real-world data collected on an arterial of the city of Salerno (south of Italy). Results showed that the estimated parameters are consistent with the literature.

Keywords: network signal setting design, cell transmission model, platoon dispersion model, calibration, validation

\footnotetext{
${ }^{1}$ Department of Civil Engineering, University of Salerno, Via Giovanni Paolo II, 132, 84084 Fisciano (SA), Italy, EU. Email: fstorani@ unisa.it. ORCID: https://orcid.org/0000-0001-6540-2658

${ }^{2}$ Department of Civil Engineering, University of Salerno, Via Giovanni Paolo II, 132, 84084 Fisciano (SA), Italy, EU. Email: rdipace @ unisa.it. ORCID: https://orcid.org/0000-0001-7589-8570

${ }^{3}$ Department of Civil Engineering, University of Salerno, Via Giovanni Paolo II, 132, 84084 Fisciano (SA), Italy, EU. Email: sdeluca@ unisa.it. ORCID: https://orcid.org/0000-0002-4597-5018

${ }^{4}$ Port Authority of Naples. Naples (NA), Italy, EU. Email: s.memoli@ porto.napoli.it.

ORCID: https://orcid.org/0000-0001-5997-7164
} 


\section{INTRODUCTION AND MOTIVATION}

Several researchers have investigated the signal setting design with regards to single junctions [1] and network optimisation [2] by the application of mono-criterion [3], and multicriteria optimisation [4]. Depending on the considered layout, whether it is a single junction or a network of interacting adjacent junctions, the considered decision variables are either the green timings with 0 cycle length or the green timings, the cycle length, and the offsets. When the optimisation is a single junction, the usually considered criteria are the capacity factor and the total delay. For a network optimisation layout, the considered criteria are commonly the delay (that is, the network total delay) and the total time spent. Additionally, these criteria can be combined to properly formulate a multi-criteria optimisation by considering the cited performance indicators and other impact indicators (or their proxy) such as emissions, fuel consumption, etc.

The focus of this paper is on the network signal setting design. However, a proper estimation of the traffic flow performances is required to optimise the decision variables. The most common phenomena to consider are queue dispersion with its horizontal propagation to obtain a realistic computation of the network Total Delay (objective function of the optimisation procedure). Thus, an enhanced traffic flow model able to reproduce these phenomena is pertinent.

Following the literature, the dispersion between successive interacting junctions is usually modelled by considering the Platoon Dispersion Model (PDM; [5]), although this model cannot simulate the horizontal queue propagation. Thus, the literature has investigated, adopting new models to address these features, primarily based on the general Lighthill-Whitham-Richards (LWR) theory. Indeed, [6] presents a numerical method for generating different degrees of dispersion of vehicle platoons or rarefaction waves using a concave fundamental diagram. Author [7] incorporates various fundamental diagrams in CTM, together with several formulas of kinematic waves, generating different types of queue dispersion and spill over.

The proposed CT and PDM model, based on the combination between the PDM and the space-time discrete Cell Transmission Model (CTM; [8]), was specified, tested, and compared against other benchmark models [9] by the authors, but not calibrated. Therefore, this paper focuses on the proper calibration and validation of the model referred to as a phenomena reproduction of flow dispersion and queue propagation, using real-world data.

This paper is organised as follows: Section II summarises the theoretical background; Section III describes the considered traffic flow model; Section IV details the case study; Section V presents the results of the calibration and validation; finally, Section VI summarises conclusions and future works.

\section{THEORETICAL BACKGROUND}

This section provides the theoretical background of the proposed model CT and PDM, starting from the description of the Platoon Dispersion Model (PDM) and the Cell Transmission Model (CTM). 


\subsection{Platoon dispersion model}

Considering an arterial network composed of two successive junctions, if a single (or submultiple) cycle length $\mathrm{C}$ is applied, the temporal flow profile repeats itself among consecutive cycles, creating a Cyclic Flow Profile (CFP).

The cyclic flow profile summarises the following relevant phenomena, which occur between the two junctions:

- the confluence of the cyclic flow profiles moving from different approaches towards the same exit of the upstream junction;

- the dispersion related to the speed of vehicles on the connecting link;

- the distortion of the cyclic flow profile at the downstream junction due to the traffic timings effect;

- the division of flow profiles from the downstream junction towards different approaches.

The platoon dispersion model calculates a downstream traffic flow from an upstream value, in discrete time steps. Let:

$\mathrm{n}$ be the length of the time step (or clock tick);

1 be the upstream junction;

$\mathrm{h}$ be the downstream junction;

$\mathrm{i}$ be the index of the time step (sub-interval of the time discretisation);

$\Delta \mathrm{i}$ be the number of time steps in which the incoming flow is dispersed;

$\mathrm{q}_{\mathrm{II}}(\mathrm{i})$ be the entering flow on the upstream junction 1, at time step $\mathrm{i}$;

$\mathrm{q}_{\mathrm{s} . \mathrm{h}}(\mathrm{i}+$ be the outflow on the downstream junction $\mathrm{h}$, at time step $\mathrm{i}+\Delta \mathrm{i}$;

$\Delta \mathrm{i})$

$t_{\mathrm{h}}$ be the travelling time, depending on the length of the connecting link, $\mathrm{l}_{\mathrm{h}}$, and the given cruise speed;

$\mathrm{q}_{\mathrm{I.I}}(\mathrm{i})$ be the entering flow at the upstream junction 1;

$\alpha$ be the platoon dispersion factor, which can vary between 0.2 and 0.5 depending on the site-specific geometric (the identified roadway conditions are

low/moderate/high friction), and traffic conditions;

$\beta$ be the travel time factor;

$\mathrm{F}$ be the smoothing factor,

The model can be rewritten and the downstream traffic flow can be computed using the Robertson platoon dispersion following a shifted geometric series. Then, the platoon dispersion effect is calculated as:

$$
q_{S, h}(\mathrm{i}+\Delta \mathrm{i})=\mathrm{F} \cdot q_{I, 1}(\mathrm{i})+(1-\mathrm{F}) \cdot q_{S, h}(\mathrm{i}+\Delta \mathrm{i}-1)
$$

with:

$$
\begin{gathered}
\Delta \mathrm{i}=\operatorname{int}\left(0.5+0.8 \cdot \frac{t_{l h}}{n}\right) \\
\mathrm{F}=\frac{1}{1+\alpha \cdot \beta \cdot t_{l h}}
\end{gathered}
$$


It is worth noting that if the distance between the junctions increases, the travelling time $\mathrm{t}_{\mathrm{h}}$ increases, then $\mathrm{F}$ approaches zero, and the cyclic flow profile becomes more uniformly distributed.

Following the literature regarding the platoon dispersion factor $\alpha$ and the travel time factor $\beta$, it is necessary to adopt different values. It has been demonstrated $[10,11])$ that the travel time factor $\beta$ is dependent on the platoon dispersion factor $\alpha$. Finally, these authors developed a specific procedure to calibrate the platoon dispersion factor $\alpha$ starting from the experienced travel times of each vehicle, while the typical procedure is to select the one that minimises the sum of squares error between the field-observed and the estimated downstream flow profiles for a given upstream flow profile. However, further studies by other authors $[12,13]$ pointed out that the procedure proposed by [11] was myopic to the effect of the time step size, and it was suitable when the time step is equal to 1 second. In particular, the alternative formulation proposed by them highlights that the three factors $\left(\alpha_{n}, \beta_{n}, F_{n}\right)$ formulated as in the following, are explicitly dependent on the size of the time interval (n).

Let:

T' be the expected travel time

$\sigma^{2}$ be the travel time variance

Then, the following expressions can be specified:

$$
\begin{gathered}
\beta_{n}=\frac{1}{1+\alpha_{n}} \\
\beta_{n}=2 T^{\prime}+n-\frac{\left(n^{2}+4 \cdot \sigma^{\prime 2}\right)^{\frac{1}{2}}}{2 T^{\prime}} \\
F_{n}=n \cdot \frac{\left[\left(n^{2}+4 \cdot \sigma^{\prime 2}\right)^{\frac{1}{2}}-n\right]}{2 \sigma^{\prime 2}}
\end{gathered}
$$

Finally, two essential findings were pointed out by [13]:

1. to provide an effective design of the signal settings of the traffic lights, proper calibration of the traffic flow model parameters is required;

2. the objective function is more sensitive to the travel time factor than the platoon dispersion factor. Therefore, the calibration procedure must focus on the travel time factor while fixing the platoon dispersion factor following the literature [14].

\subsection{Macroscopic traffic flow modelling}

The macroscopic traffic flow models can be classified in:

- space discrete models,

- space continuous models.

Space discrete models describe the propagation of flows through a link by the relationships between whole link variables, such as link travel time, link inflows, outflows, or link volume (that is, the number of vehicles on the link) at each point in time. These models do not require any space discretisation, and thus, are also named link-based [15-17]. 
The continuous space models derive from the analogy between vehicular flow and flow of continuous media (for example, fluids or gases), yielding flow models with a limited number of partial differential equations that allow the description of the dynamics of variables as the following:

- density $(k)$ : Typical variable from physics adopted by traffic science to express the number of vehicles per kilometre of road,

- flow rate $(q)$ : Represents the number of vehicles that passes a cross-section per time unit,

- mean speed $(u)$ : Defined as the quotient of the flow rate and the density.

By describing the propagation of the flow states in every space-time point of the link, these models require a dense space discretisation, and thus, are also named point-based models. The most straightforward continuous traffic flow model is the first-order model developed concurrently by Lighthill et al. $[18,19]$, based on the assumption that the number of vehicles is conserved between any two points if there are no entrances (sources) or exits (sinks). The result is a continuous model known as the Lighthill-Whitham-Richards (LWR) model, given as:

$$
\frac{\partial k(s, t)}{\partial t}+\frac{\partial q(s, t)}{\partial s}=0
$$

The flow $\mathrm{q}$ is further related to the density $\mathrm{K}$ and the mean speed $\mathrm{u}$ from the relation:

$$
q(s, t)=K(s, t) \cdot u(s, t)
$$

The mean stream speed can be expressed as a decreasing function of the density $k$ (traffic fundamental diagram), and according to the above equation, a relationship between flow $q$ and density $k$ is given from:

$$
q(s, t)=q(k(s, t), s)
$$

So that the LWR model may be expressed as:

$$
\frac{\partial k(s, t)}{\partial t}+q^{\prime}(k) \cdot \frac{\partial k(s, t)}{\partial s}=0
$$

where:

$$
q^{\prime}(k)=\frac{\partial q}{\partial k}
$$

When vehicles are not impeded by other traffic, they travel at a maximum speed of $u_{f}$ (freeflow speed). On saturated roads, flow rate and speed are down to zero. The vehicles are queuing and there is a maximum density of $k_{j}$ (jam density). The capacity of a road is equal to the maximum flow rate $q_{c}$. The maximum flow rate of $q_{c}$ has an associated capacity speed of $u_{c}$ and a capacity density of $k_{c}$.

As the differential equations used in LWR models are difficult to solve accurately, especially for high-density variations as bottlenecking (in this case, the LWR calls for a shock wave), different approximative techniques were proposed to solve the equations. Newell [20] introduced a simplified theory of kinematic waves to predict the state of flow at an extreme 
depending on the traffic conditions of another one, without considering the traffic condition at the intermediate sections, by using cumulative inflow and outflow curves. This theory relates traffic flow $q$ with density $k$, following a triangular-shaped fundamental diagram. In this way, the author proposed a space-discrete model (link-based), which provides link travel times complying with the simplified kinematic wave theory. In this equilibrium relation, the mean speed equals the maximum speed for all traffic states with densities smaller than the critical capacity density. The side of the triangle that describes the saturated capacity state has a negative constant slope $\mathrm{w}$ (that is, the wave speed).

Another way of solving the LWR space-continuous problem was introduced by Daganzo [8] through the "Cell Transmission Model", developed as a discrete analogue of the LWR differential equations in the form of finite-difference equations. In a cell-transmission scheme, a road is divided into small sections (cells), and the model tracks the content of each cell (number of vehicles or density) as time passes. This value is updated at closely spaced instants (time steps or clock ticks) by calculating the number of vehicles that cross the boundary separating each pair of adjoining cells at each time step. It should be pointed out that the relation between the cell length and the time step has to comply with the Courant-Friedrich-Lewy condition $\left(u_{f} \cdot \Delta t \leq \Delta x\right)$ for the stability of explicit solution methods.

Let:

$\Delta x$ be the cell length;

$\Delta t$ be the time step;

$u_{f}$ be the free-flow speed;

$\omega$ be the shock wave speed in congested traffic;

$k_{j}$ be the jam density;

$Q_{i}$ be the maximum flow rate in cell $i$;

$k_{i}(t)$ be the density in cell $i$ at time $t$

$n_{i}(t)$ be the number of vehicles in cell $i$ at time $t$, equal to $k_{i}(t) \cdot \Delta x$;

$N_{i}$ be the maximum number of vehicles in cell $i$, equal to $k_{j} \cdot \Delta x$;

$Y_{i}(t)$ be the average flow exiting the boundary of cell $i$ at time $t$.

The average flow exiting the boundary of cell $i$ from time step $t$ to time step $t+1$ is given by:

$$
Y_{i}(t)=\min \left(u_{f} \cdot k_{i}(t), \min \left(Q_{i}, Q_{i+1}\right), \omega \cdot\left(k_{j}-k_{i+1}(t)\right)\right)
$$

Or, if the cell has a length equal to the free-flow speed times the time step $\left(\Delta x=u_{f} \cdot \Delta t\right)$, the equation can be rewritten depending on the number of vehicles, as:

$$
Y_{i}(t)=\min \left(\frac{n_{i}(t)}{\Delta t}, \min \left(Q_{i}, Q_{i+1}\right), \frac{\omega}{u_{f}} \cdot \frac{\left(N_{i+1}-n_{i+1}(t)\right)}{\Delta t}\right)
$$

Which is the result of a comparison between the maximum flow of vehicles that can be "sent" by the cell $i$ directly upstream of the boundary during the time step:

$$
S_{i}(t)=\min \left(Q_{i}, u_{f} \cdot k_{i}(t)\right)
$$

and those that can be "received" by the downstream cell i+1: 


$$
R_{i+1}(t)=\min \left(Q_{i+1}, \frac{\omega}{u_{f}} \cdot \frac{\left(N_{i+1}-n_{i+1}(t)\right)}{\Delta t}\right)
$$

Hence, the flow $Y_{i}(t)$ can be rewritten as:

$$
Y_{i}(t)=\min \left(S_{i}(t), R_{i+1}(t)\right)
$$

Lastly, the density $k_{i}(t+1)$ of cell $i$ is updated with the exiting flow $Y_{i}(t)$ of cell $i$ and the incoming flow $Y_{i-1}(t)$ from the boundary of the upstream cell $i-1$ as:

$$
k_{i}(t+1)=k_{i}(t)+\left[Y_{i-1}(t)-Y_{i}(t)\right] \cdot \frac{\Delta t}{\Delta x}
$$

Daganzo's CTM cannot fully predict a realistic traffic flow behaviour due to the assumption of uniform speed: platoons keep the same density between the upstream and the downstream stop-line section, and all vehicles travel at the same free-flow speed. Thus, a traffic flow model that considers platoon dispersion was proposed [9]. This model uses a Drake speed-density relationship [21], validated against real-world data collected on a signalised arterial site in Salerno (Italy). The following sections show the results of this combination. In future works, further mono-regime [22-25] or two regimes [26] speed-density relationships could be adopted to attempt the best fit with field data.

\section{PROPOSED CELL TRANSMISSION AND PLATOON DISPERSION MODEL (CT and PDM)}

As shown in the CTM equations, there is no platoon dispersion due to the assumption that all vehicles travel at the same speed (therefore maintaining the same density) when moving towards the downstream section. Thus, to overcome this shortfall, the flow propagation is modelled applying at each cell, the well-known Drake speed-density relationship, in which the corresponding flow, $X_{i}(t)$, is computed as follows:

$$
X_{i}(t)=k_{i}(t) \cdot u_{f} \cdot \exp \cdot\left(-0.5\left(\frac{k_{i}(t)+k_{i+1}(t)}{2 \cdot k_{m}}\right)^{2}\right)
$$

where:

$u_{f}$ is the free-flow speed;

$k_{m}$ is the traffic density at maximum flow;

$k_{i}(t)$ is the density in cell $i$ at time $t$;

$k_{i+1}(t)$ is the density in cell $i+1$ at time $t$;

Next, the flow $Y_{i}(t)$ to the adjacent downstream cell is formulated as:

$$
Y_{i}(t)=\min \left(S_{i}(t), R_{i+1}(t), X_{i}(t)\right)
$$




\section{CASE STUDY}

A field study was identified, providing empirical support to test the proposed traffic flow model via its traffic-dispersion patterns. The purpose of the survey and subsequent analysis was to measure the extent to which the field-observed and simulated traffic stream characteristics (obtained through the CT and PDM described in section III) match each other.

The study uses data from a signalised arterial of Salerno (Italy) (Figure 1). It has $520 \mathrm{~m}$ in length and contains two signalised junctions with a cycle of 80 seconds. The observation of the traffic characteristics was in three different sections:

- at the upstream signalised intersection, considering only the straight-on stream of traffic,

- in the middle section, $240 \mathrm{~m}$ from the upstream junction,

- at the signalised downstream intersection.

The traffic flow data were collected for one hour, on the morning peak hour (from 7:30 a.m. to 8:30 a.m.). Figure 1 indicates the layout of the arterial and the study sections.

This study compares the queue propagation and platoon dispersion between the observed in the test site and the modelled using the CT and PDM model. Therefore, to calibrate the model, different procedures were applied to analyse the phenomenon of:

- platoon dispersion: matching the cyclic flow profiles detected, with the simulated in the middle section of the arterial (Figure 3),

- queue propagation: matching the uniform queue graphs at the downstream signalised junction (Figure 4) of the field-observed and the simulated arrivals.

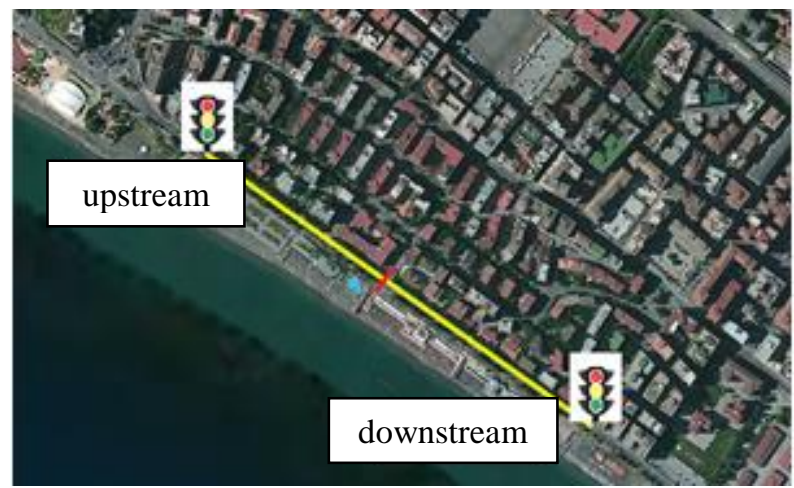

Fig. 1. Layout of the arterial and study sections. Google, (n.d.) Screenshot of the city of Salerno Source: Google Maps

Individual vehicles were scanned and recorded at chosen intervals (time steps/clock tick) of 1 second, using six loop detectors placed on the roadway, two at each section: two of them were located within $5 \mathrm{~m}$ of the junction with respect to the downstream signalised junction, two of them were located within $5 \mathrm{~m}$ of the junction with respect to the upstream signalised junction, and the last couple of 2 detectors was placed in the middle of the arterial.

The speed and cell length adopted for the simulation was kept constant. Considering a time step $\mathrm{t}=1$ second, 1/3600th of an hour, and identified a free-flow speed, $\mathrm{v}=50 \mathrm{kmph}$, the minimum cell length equal to the maximum distance travelled by a vehicle in free-flow speed during one clock tick $=\mathrm{v} \times \mathrm{t}=50 \times(1 / 3600) \approx 1 / 72 \mathrm{~km}$. Since the arterial has $0.560 \mathrm{~km}$ of 
length, then the number of cells is $0.560 /(1 / 72) \approx 40$ cells. Fixed a jam density of $167 \mathrm{veh} / \mathrm{km}$, the maximum number of vehicles that a cell can hold, $\mathrm{N}$, equal to cell length $\mathrm{x}$ jam density is $167 \times(1 / 72)=2.32$ vehicles. Fixing a road capacity $q_{\max }$ of $3600 \mathrm{veh} / \mathrm{hr}$, the maximum number of vehicles that can flow into cell $i$ from time $t$ to $t+1, Q$, results $3600 \times(1 / 3600)=1$ vehicles.

Given the input flow $q_{i}$ moving from the upstream junction during the cycle length, the incoming flow $y_{0}(t)$ to the first cell of the link has been set equal to the desired link input flow (constrained by the capacity of the approach) for the corresponding time interval (CFP of Figure 2).

The collected data were aggregated in four main sub-intervals, each one with a duration of 15 minutes. The vehicles were classified depending on their category (for example, motorcycle, car, heavy vehicles, etc.). Finally, to deal with the heterogeneity in a mixed traffic situation, these categories were combined in terms of passenger car unit (PCU) by applying a relative weight factor to the traffic volume of each vehicle category. In this way, the flow $q_{k, i-i+T}$ was obtained for each sub-interval of 15 minutes during one hour of simulation, considering the approach $k$ in the sub-interval from $i$ to $i+T$. Then, with the following expressions, the peak hour factor and the mean value of the flow for the simulation interval are computed:

$$
\begin{gathered}
\operatorname{PHF}_{k}=\frac{q_{\mathrm{k}, 0-15}+q_{\mathrm{k}, 15-30}+q_{\mathrm{k}, 30-45}+q_{\mathrm{k}, 45-60}}{4 \max \left(q_{\mathrm{k}, 0-15} ; q_{\mathrm{k}, 15-30} ; q_{\mathrm{k}, 30-45} ; q_{\mathrm{k}, 45-60}\right)} \\
q_{\mathrm{k}}=\frac{\left(q_{\mathrm{k}, 0-15}+q_{\mathrm{k}, 15-30}+q_{\mathrm{k}, 30-45}+q_{\mathrm{k}, 45-60}\right)}{\mathrm{PHF}_{k}} \\
q_{\mathrm{k}}=4 \max \left(q_{\mathrm{k}, 0-15} ; q_{\mathrm{k}, 15-30} ; q_{\mathrm{k}, 30-45} ; q_{\mathrm{k}, 45-60}\right)
\end{gathered}
$$

Below, Table 1 summarises the characteristics of the arterial, while Table 2 reports the time and spatial discretisation adopted in the CT and PDM together with the Q and N values.

Tab. 1

Model characteristics

\begin{tabular}{ccccc}
\hline $\begin{array}{c}\text { Free-flow speed } \\
u_{f}[\mathrm{~km} / \mathrm{h}]\end{array}$ & $\begin{array}{c}\text { Road length } \\
L[\mathrm{~km}]\end{array}$ & $\begin{array}{c}\text { Jam density } \\
k_{j}[\mathrm{veh} / \mathrm{km}]\end{array}$ & $\begin{array}{c}\text { Max flow rate } \\
Q_{\max }[\mathrm{veh} / \mathrm{h}]\end{array}$ & $\begin{array}{c}\text { Input flow } \\
Y_{1}[\mathrm{veh} / \mathrm{h}]\end{array}$ \\
\hline 50 & 0.56 & 167 & 3600 & 1725 \\
\hline \hline
\end{tabular}

Tab. 2

Spatial and time discretisation features adopted in CT and PDM.

\begin{tabular}{cccc}
\hline $\begin{array}{c}\text { Clock tick } \\
\Delta t[\mathrm{~s}]\end{array}$ & $\begin{array}{c}\text { Cell length } \\
\Delta x[\mathrm{~m}]\end{array}$ & Number of cells & $\begin{array}{c}\text { Maximum number of vehicles per cell } \\
\mathrm{N}[\mathrm{veh}]\end{array}$ \\
\hline 1 & 13.9 & 40 & 2.32 \\
\hline \hline
\end{tabular}

\section{MODEL CALIBRATION AND VALIDATION}

This section presents the details of the model calibration and validation. Subsequently, an aggregate goodness-of-fit measurement quantifies the degree to which the model results fit 
the field data. The parameters to calibrate are the platoon dispersion factor $\alpha$ and the travel time factor $\beta$.

A first analysis confirmed the two main findings pointed out by [13], which refers to the necessity to carry out a proper calibration of the traffic flow model parameters and the identification of their sensitivity. Indeed, the objective function is more sensitive to the travel time factor than the platoon dispersion factor. Therefore, following the literature [14], the platoon dispersion $\alpha$ was fixed to 0.25 ; and the travel time parameter $\beta$ remains variable to calibrate, Comparing the measured flow volume in the middle section of the arterial and the one simulated in the proposed CT and PDM.

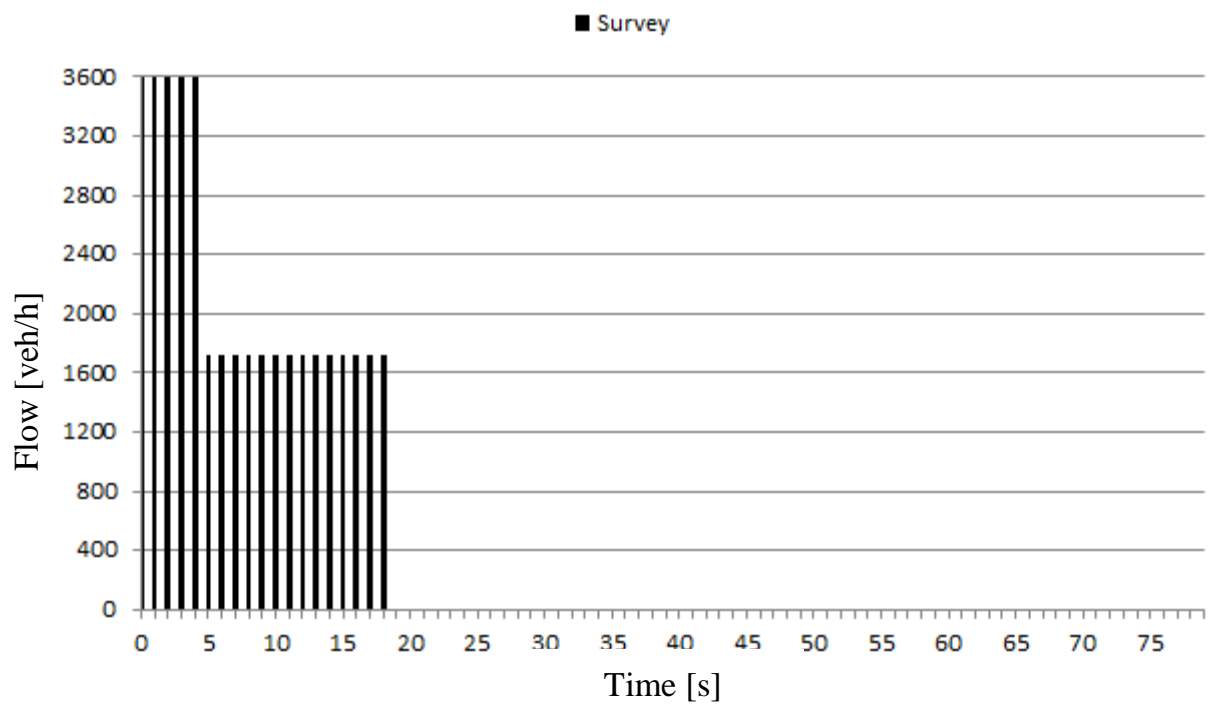

Fig. 2. Upstream junction CFP

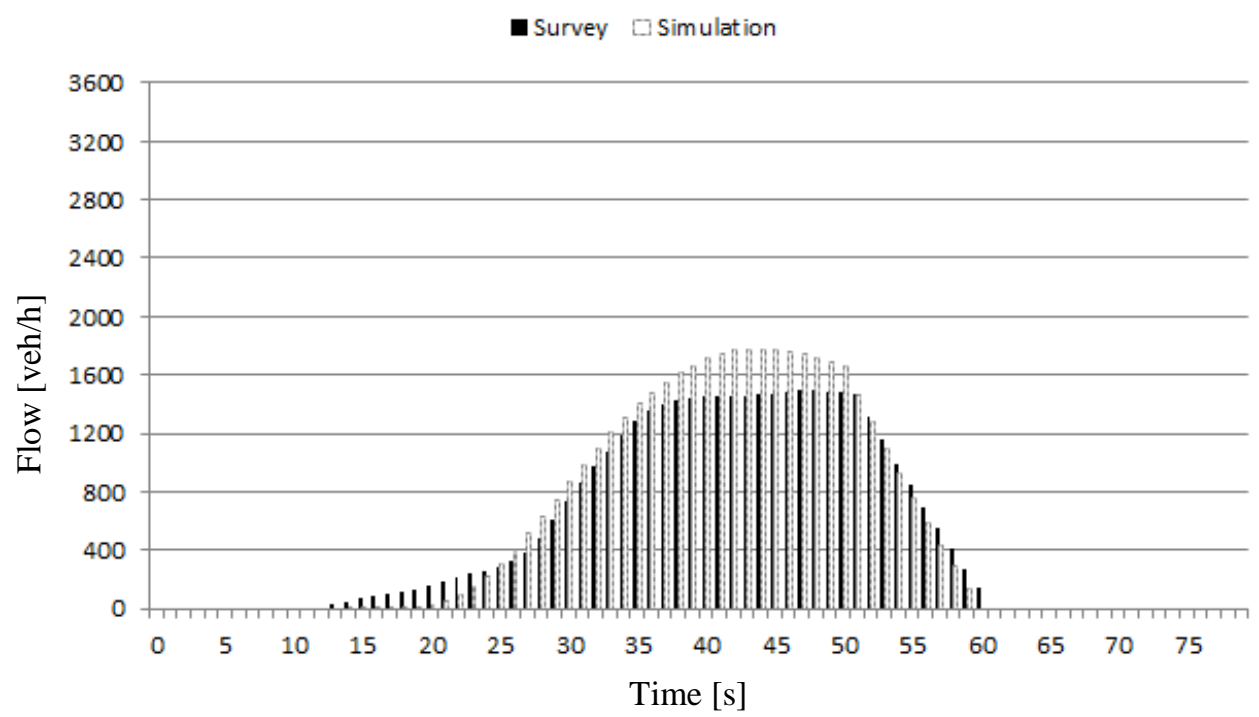

Fig. 3. Middle section CFP

The term "aggregate" refers to the combination of all the measurements into a single metric. The aggregate measure adopted therein is the Mean Absolute Proportional Error (MAPE), calculated as follows: 


$$
\operatorname{MAPE}=\left(\frac{1}{\mathrm{~m}}\right) \sum \mathrm{i}=1 \ldots \mathrm{m} \frac{|\mathrm{Oi}-\mathrm{Ei}|}{\mathrm{Oi}}
$$

where:

Oi is the measure of performance observed from field data,

Ei is the measure of performance estimated by the simulation model.

The MAPE estimates error as a proportion of the observed mean, making it easily understandable due to its physical meaning.

The queue length is estimated from the characteristics of the interacting approach at the downstream signalised junction (cycle-length $80 \mathrm{~s}$, effective green $45 \mathrm{~s}$ ) to then compare its values with the ground truth data.

The MAPE computation returns a value indicating that the simulation and the modelled results differ by $14 \%$. Finally, the travel time factor $\beta$, estimated was equal to 0.84 .

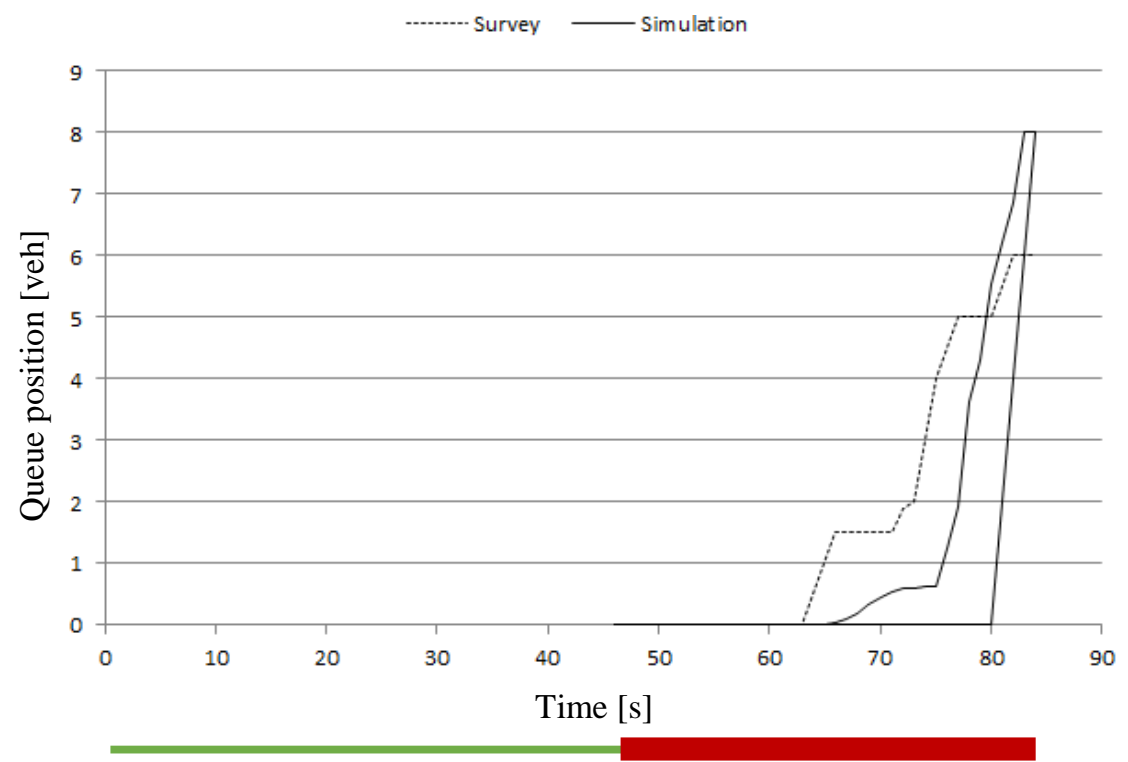

Fig. 4. Uniform queue graph at downstream junction

\section{CONCLUSION AND FUTURE WORKS}

In the case of network signal setting design, reliable traffic flow modelling is required to simulate the vehicles dispersion and the horizontal queue propagation. Thus, this paper proposes a macroscopic traffic flow model combining the Cell Transmission and the Platoon Dispersion model. This paper aims to calibrate and validate the model by considering the realworld data collected in the city of Salerno (in the south of Italy).

The MAPE indicator was adopted and the overall results return that the consistency between the observed and the simulated results is around $14 \%$. In terms of calibration consistently with [13], the travel time factor $\beta$ was estimated and it was equal to 0.84 .

For future works, it would be worthy of interest:

- to extend the model validation by considering data at a network level,

- to analyse other flow-density relationships,

- to include in the proposed model, the simulation of multi-commodity flow sources. 


\section{Acknowledgements}

This research was partially supported by the University of Salerno, under the $\mathrm{PhD}$ programme on transportation (Ph.D. School in Environmental Engineering), local grant n. ORSA180377- 2018, local grant n. ORSA191831- 2019.

\section{References}

1. Cantarella G.E., G. Improta. 1988. „Capacity factor or cycle time optimization for signalized junctions: A graph theory approach". Transportation Research Part B: Methodological 22(1): 1-23. DOI: 10.1016/0191-2615(88)90031-8.

2. Di Gangi Massimo, Giulio E. Cantarella, Roberta Di Pace, Silvio Memoli. 2016. „Network traffic control based on a mesoscopic dynamic flow model”. Transportation Research Part C: Emerging Technologies 66: 3-26. DOI: 10.1016/j.trc.2015.10.002.

3. Cantarella Giulio Erberto, Stefano De Luca, Roberta Di Pace, Silvio Memoli. 2014. „Signal Setting Design at a Single Junction Through the Application of Genetic Algorithms". Advances in Intelligent Systems and Computing: 321-331.

4. Cantarella Giulio Erberto, Stefano De Luca, Roberta Di Pace, Silvio Memoli. 2013. The Application of Multicriteria Genetic Algorithms for Signal Setting Design at a Single Junction”. In: 2013 8th EUROSIM Congress on Modelling and Simulation. IEEE, 472-477. ISBN: 978-0-7695-5073-2.

5. Robertson DI. 1969. „TRANSYT: A Traffic Network Study Tool”. Road Research Laboratory. UK.

6. Geroliminis Nikolaos, Alexander Skabardonis. 2005. „Prediction of Arrival Profiles and Queue Lengths Along Signalized Arterials by Using a Markov Decision Process". Transportation Research Record: Journal of the Transportation Research Board 1934: 116-124. DOI: 10.3141/1934-12.

7. Chow Andy H.F., Shuai Li, W.Y. Szeto, David Z.W. Wang. 2015. „Modelling urban traffic dynamics based upon the variational formulation of kinematic waves". Transportmetrica B: Transport Dynamics 3(3): 169-191. DOI: $10.1080 / 21680566.2015 .1005559$.

8. Daganzo Carlos F. 1995. „The cell transmission model, part II: Network traffic”. Transportation Research Part B: Methodological 29(2): 79-93. DOI: 10.1016/0191-2615(94)00022-R.

9. Cantarella Giulio E., Stefano De Luca, Roberta Di Pace, Silvio Memoli. 2015. „Network Signal Setting Design: Meta-heuristic optimisation methods". Transportation Research Part C: Emerging Technologies 55: 24-45. DOI: 10.1016/j.trc.2015.03.032.

10. Yu L., M. Van Aerde. 1995. „Implementing TRANSYT's macroscopic platoon dispersion in microscopic traffic simulation models". In: 74thTransportation Research Board Annual Meeting. Washington DC, USA.

11. Yu Lei. 2000. „Calibration of Platoon Dispersion Parameters on the Basis of Link Travel Time Statistics". Transportation Research Record: Journal of the Transportation Research Board 1727(1): 89-94. DOI: 10.3141/1727-11.

12. Rakha Hesham, Mohamadreza Farzaneh. 2006. „Issues and Solutions to Macroscopic Traffic Dispersion Modeling”. Journal of Transportation Engineering 132(7): 555-564. DOI: 10.1061/(ASCE)0733-947X(2006)132:7(555). 
13. Farzaneh Mohamadreza, Hesham Rakha. 2006. „Procedures for Calibrating TRANSYT Platoon Dispersion Model". Journal of Transportation Engineering 132(7): 548-554. DOI: 10.1061/(ASCE)0733-947X(2006)132:7(548).

14. Retzko Hans-Georg, M Schenk. 1993. „Effects of the platoon dispersion on the optimizing of fixed-time signal control in road networks". Transportation and Traffic Theory: Proc., 12th Int. Symp. on the Theory of Traffic Flow and Transportation. P. 539-551.

15. Astarita Vittorio. 1996. „Flow propagation description in dynamic network loading models". In: Proceedings of the International Conference on Applications of Advanced Technologies in Transportation Engineering. P. 599-603.

16. Ran Bin, David Boyce 1996. Modeling Dynamic Transportation Networks. Berlin, Heidelberg: Springer Berlin Heidelberg. ISBN: 978-3-642-80232-4.

17. Wu J.H., Y. Chen, M. Florian. 1998. „The continuous dynamic network loading problem: a mathematical formulation and solution method". Transportation Research Part B: Methodological 32(3): 173-187. DOI: 10.1016/S0191-2615(97)00023-4.

18. Lighthill M.J., G.B. Whitham. 1955. „On kinematic waves II. A theory of traffic flow on long crowded roads". Proceedings of the Royal Society of London. Series A.

Mathematical and Physical Sciences 229(1178): 317-345. DOI: 10.1098/rspa.1955.0089.

19. Richards Paul I. 1956. „Shock Waves on the Highway”. Operations Research 4(1): 42-51. DOI: 10.1287/opre.4.1.42.

20. Newell G.F. 1993. „A simplified theory of kinematic waves in highway traffic I: General theory. II: Queuing at freeway bottlenecks. III: Multi-destination flows". Transportation Research Part B P. 281-313.

21. Drake Joseph S., Joseph L. Schofer, Adolf D. May Jr. 1967. „A Statistical Analysis of Speed Density Hypotheses". In: Third International Symposium on the Theory of Traffic Flow Proceedings. New York: Elsevier North Holland, Inc.

22. Greenberg Harold. 1959. „An Analysis of Traffic Flow”. Operations Research 7(1): 79-85. DOI: 10.1287/opre.7.1.79.

23. Underwood R.T. 1961. „Speed, volume, and density relationships”. In: Qualty and Theory of Traffic Flow. A Symposium: 141-188. New Haven, Connecticut: Highway Traffic.

24. Drew Donald R. 1968. Traffic flow theory and control. New York, McGraw-Hill.

25. Pipes Louis A. 1967. „Car following models and the fundamental diagram of road traffic”. Transportation Research 1(1): 21-29. DOI: 10.1016/0041-1647(67)90092-5.

26. Edie Leslie C. 1961. „Car-following and steady-state theory for noncongested traffic”. Operations research 9(1): 66-76.

Received 02.10.2021; accepted in revised form 08.12.2021

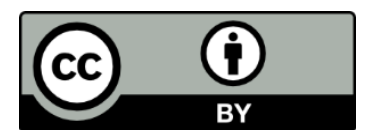

Scientific Journal of Silesian University of Technology. Series Transport is licensed under a Creative Commons Attribution 4.0 International License 